\title{
PERFORMANCE OF ISOTROPIC MAGNETORHEOLOGICAL RUBBER MATERIALS
}

Mattias Lokander

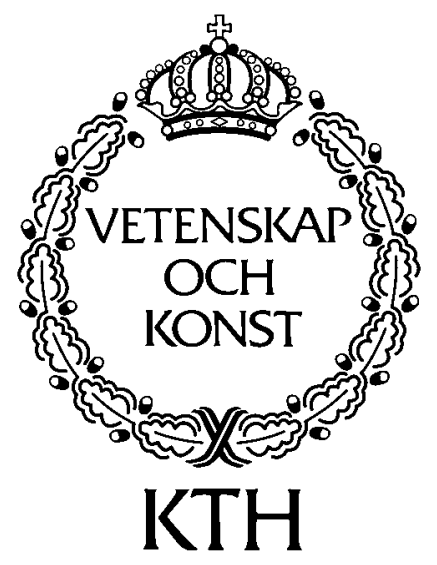

Department of Fibre and Polymer Technology

Royal Institute of Technology

Stockholm 2002 


\begin{abstract}
Magnetorheological (MR) rubber materials are controllable composites that consist of magnetically polarisable particles in a rubber matrix. They belong to a group of socalled "smart materials" that have seen an increased interest recently. These materials have rheological properties that can be changed continuously, rapidly and reversibly by an applied magnetic field. With suitable control algorithms and solid state electronics, they can respond to changes in their environment. Although few applications of these materials have yet been reported in the literature, the possibilities for materials with controllable stiffness are numerous. Examples of potential applications are tuned vibration absorbers, and stiffness-tuneable mounts and suspensions.
\end{abstract}

The purpose of this work is to increase our knowledge relating to magnetorheological materials for damping applications, using construction rubber as the matrix. The materials should exhibit large responses to an applied magnetic field and have good mechanical properties. In order to simplify their manufacture, the use of a magnetic field during production should, if possible, be avoided.

MR rubber materials were made from nitrile and natural rubber, and irregularly shaped iron particles several micrometers in size. The particles were not aligned by a magnetic field prior to the vulcanisation; hence, the materials can be considered to be isotropic. These materials show a large MR effect, e.g. an increase in the shear modulus when a magnetic field is applied, although the particles are not aligned within the material. This is explained by the low critical particle volume concentration (CPVC) of such particles. Similar behaviour can be obtained with materials containing carbonyl iron, if the particles are aggregated and thereby behave like large irregular particles. The iron particle concentration has to be very close to the CPVC in order to obtain a large MR effect.

The absolute MR effect in isotropic MR rubber materials with large irregular iron particles is independent of the matrix material, and the relative MR effect can thus be increased by the addition of plasticisers. Other ways of increasing the MR effect are to increase the strength of the magnetic field, although the materials saturate magnetically at high field strengths, or to use small strain amplitudes. The strong strain amplitude dependence of the MR effect suggests that isotropic MR rubber materials are most suitable for low amplitude applications, such as sound and vibration insulation. Measurements at frequencies within the audible frequency range show that this is a promising application for isotropic MR rubber materials. 


\section{Table of Contents}

1. Introduction

1.1 Purpose of the Study ___ 2

1.2 MR- and ER-fluids __ 2

1.3 MR Solids __ 4

1.4 Particles _ 5

2. Experimental __ 6

2.1 Materials _ 6

2.2 Methods

2.2.1MR measurements

2.2.2 High frequency MR measurements___ 9

2.2.3 Density measurements ___ 9

2.2.4 Tensile test 10

2.2.5 Rheometer measurements 10

3. Results and discussion 11

3.1 Validity of the MR measurements

3.2 Critical Particle Volume Concentration (CPVC)

3.3 Increasing the MR Effect

3.4 MR Effect within the Audible Frequency Range___ 22

4. Conclusions — 25

5. Suggestions for Future Work

6. Acknowledgement _ 27

7. References 


\section{Introduction}

\subsection{Purpose of the Study}

Magnetorheological rubber materials are controllable composites that consist of magnetically polarisable particles in a rubber matrix. They belong to a group of so-called "smart materials" that have seen an increased interest recently. These materials have rheological properties that can be changed continuously, rapidly and reversibly by an applied magnetic field. With suitable control algorithms and solid state electronics, they can respond to changes in their environment. Although few applications of these materials have yet been reported in the literature, the possibilities for materials with controllable stiffness are numerous. Examples of potential applications are tuned vibration absorbers, and stiffness-tuneable mounts and suspensions.

The purpose of this work is to increase our knowledge relating to magnetorheological materials for damping applications, using construction rubber as the matrix. The materials should exhibit large responses to an applied magnetic field and have good mechanical properties. In order to simplify their manufacture, the use of a magnetic field during production should, if possible, be avoided.

\subsection{MR- and ER-fluids}

The idea of changing the rheological properties of materials by applying a field is not new. In the late $19^{\text {th }}$ century, it was observed that when an electric field was applied across a mixture of glycerin, paraffin and castor oil, there was a small and reversible change in viscosity. Winslow discovered a similar but larger effect using powdered oil dispersions in 1947 [1]. Winslow called the fluids "electro-viscous"[2], but the term "electrorheological" (ER) was later considered to be more appropriate to describe the behavior of the materials in an electric field. At about the same time, Rabinow discovered a similar effect of an applied magnetic field, which he named magnetorheology (MR) [3]. During the 1950's, many patents based on these phenomena, especially MR, were published. However, none of them had any commercial success due to the small difference between the activated and non-activated states of the materials, coupled with sedimentation problems. Since the difficulties were larger than was first expected, the interest in both ER and MR decreased. In the 1980's, however, with new faster generations of computers, new applications, such as controllable dampers were made possible, and again the field of ER and MR seemed interesting [4]. The possible applications of ER and MR fluids are numerous. For example, clutches, brakes, dampers and shock absorbers have been suggested for both ER- and MR-fluids [5].

The renewed interest was focused mainly on ER fluids. At this time, ER fluids were thought to be the ones that would be easiest to develop into commercial applications. One reason was that the particles in MR fluids are large and heavy, so that sedimentation is a problem. Furthermore, MR fluids have a natural limitation in strength when the saturation magnetization of the particles in the fluid is reached; hence, it was long believed that ER fluids would be developed with larger effect than MR fluids. Another reason was that 
electricity was considered to be easier to use than magnetism in the theoretical modeling of the material systems [6]. During the last decade, however, interest has once more shifted in focus since MR materials have been more successful in commercial applications. The reasons for this are that MR fluids are some order of magnitude stronger than ER fluids, and that the required field levels are easier to achieve in MR devices than in ER applications. There are several commercial applications using MR fluids, i.e. a seat damper for heavy trucks and a smooth brake for exercise bikes, but none using ER fluids [4].

ER and MR fluids are suspensions of polarisable particles in a carrier fluid. When an electric or magnetic field is applied, the particles polarize and the resulting dipoles cause the particles to form a columnar structure in the direction of the applied field. These chain-like structures restrict the motion of the fluid, so that the viscous characteristics of the suspension are increased. When the field is withdrawn, the fluid immediately returns to its original state. Both the activation and the deactivation of the fluids are completed within milliseconds after the field has been turned on or off [5].

Although there is an increase in apparent viscosity from a macroscopic point of view, the actual plastic viscosity, defined as the change in stress per unit change in shear strain, is approximately constant as the field is varied (Figure 1.1). In the absence of a field, the fluids behave approximately as Newtonian fluids. When the field is applied, they behave as Bingham bodies [5]. The yield stress $\left(\tau_{\mathrm{y}}\right)$ of the Bingham bodies, which is fielddependent, is the most important property of both MR and ER fluids. The magnetic field required to activate an MR fluid is of the order of magnitude of tenths of Tesla [5] (which may be achieved using an ordinary $12 \mathrm{~V}$ battery if the magnetic circuit is properly designed). The corresponding electric field to activate an ER fluid is of the order of magnitude of $\mathrm{kV}$, which requires high voltage equipment [7].
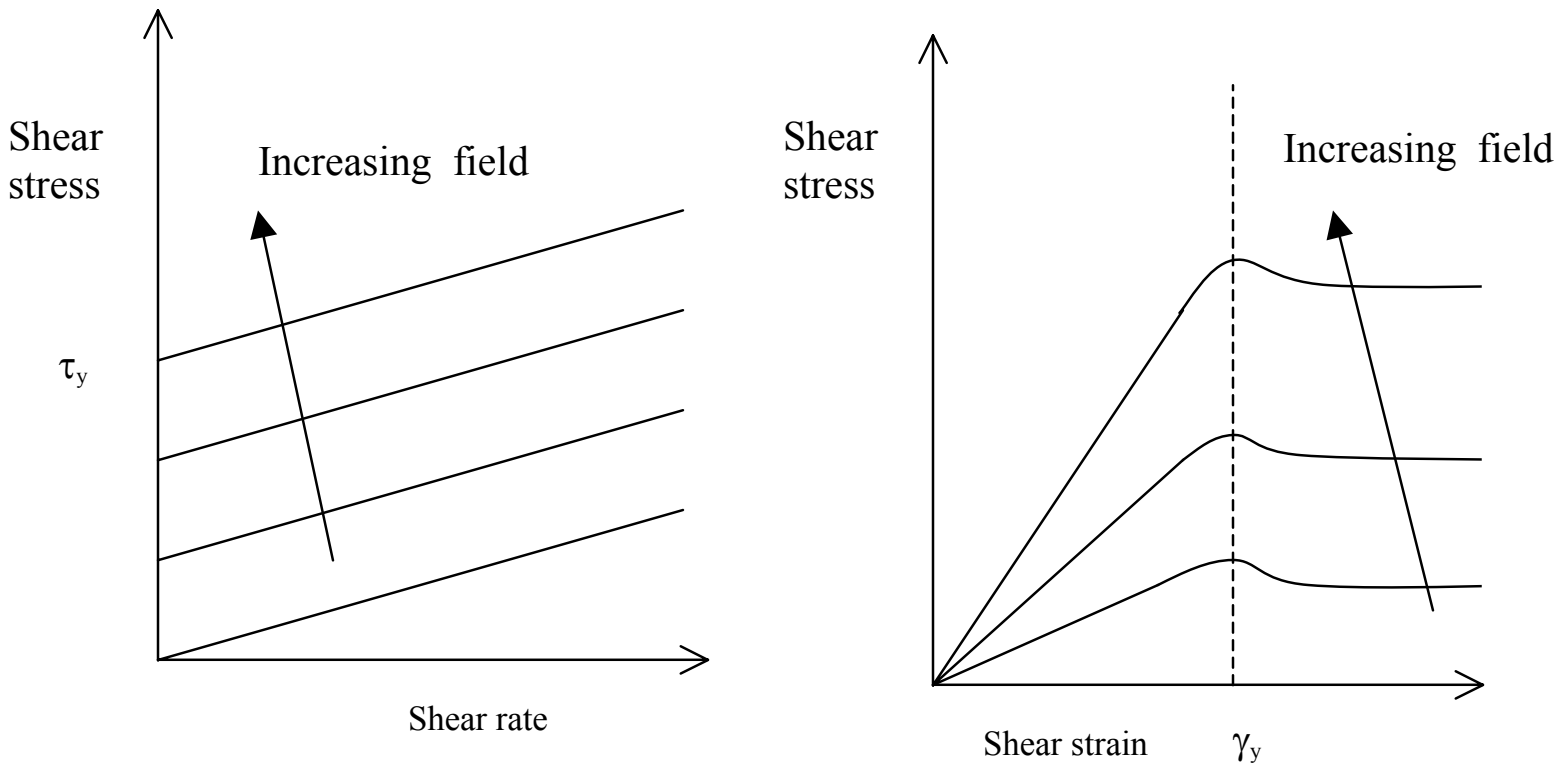

Fig. 1.1. Idealized constitutive shear behavior of MR and ER fluids 
There are some drawbacks in the use of fluids. A container is required to keep the fluid in place, and the suspended particles cause abrasion on seals and the container.

Furthermore, it is difficult to avoid the problem of sedimentation and aggregation of the particles. There are ways of solving this problem, for example by adding thixotropic agents, but such "stable" fluids have a very high zero-field viscosity instead. A recently recognized problem for MR fluids is "in use thickening". In use, MR fluids show an increase in modulus after some 100000 cycles of mechanical deformation [8]. This phenomenon is explained by abrasion of the suspended particles, which results in nanosized particles in the oil which increase the viscosity of the fluid.

\subsection{MR Solids}

MR solids are the solid analogue of MR fluids, where the matrix oils have been replaced by a crosslinked material such as a rubber or a gel. The most obvious advantage of an MR solid is that the particles are not able to settle, since the matrix is crosslinked. Furthermore, no container is needed to keep the material in place, and the MR effect may be slightly faster than in MR fluids since the particle does not move within the matrix. The latter is probably of minor importance since the MR effect in a fluids is also fast. However, MR solids work only in the pre-yield region, whereas MR fluids typically work in the post-yield state, and this makes the two groups of materials complementary rather than competitive. Thus, MR solids have a controllable, field-dependent modulus, whereas MR fluids have a field-dependent yield stress [5].

The MR effect in an MR solid is, as in an MR fluid, caused by the interaction of the induced dipoles, created by the polarization of the particles by the applied field. However, the particles in the solid matrix are not able to align in a magnetic field into the chain-like structures found in the fluids. Alignment of the particles results in a greater MR effect, but this alignment has to be created before the curing of the solid MR materials. The easiest way to create the alignment is to apply a magnetic field before the curing, when the matrix is in a more or less liquid state, and the chain-like structures are formed as in the MR fluids. The material is then cured and the particles are locked into their new positions [5].

MR solids, where magnetically polarisable particles are dispersed in an elastomer or a gel prior to the curing, have been investigated for some time. However, interest in their MR properties, i.e. a reversible change in shear modulus caused by an applied magnetic field, is quite recent. Rigbi and Jilkén [9] studied changes in the magnetic induction of ferritefilled natural rubber, caused by mechanical deformation of the material as early as 1983 . Nevertheless, it was not until the mid 1990's, when Shiga et al. replaced the semiconducting particles in their ER elastomer[10] with magnetically polarisable particles[11], that the MR effect of MR elastomers was reported. One result of the early work on elastomers filled with magnetically polarisable particles was the possibility of 
aligning particles by a magnetic field, although the particles are spherical [12]. Soft elastomers with particles aligned by a magnetic field before curing have a conductivity that is pressure-sensitive, and they can be used for touch-screen panels [13]. Another application for elastomers with dispersed magnetic particles is microwave absorption [14$16]$.

The work on MR solids, where the interest has been in the MR effect, has primarily been performed by three groups: Shiga et al. at Toyota [11], Jolly et al. at Lord Co [17, 18], and Ginder et al. at Ford [19, 20]. All these groups started working with ER and MR fluids, and then moved on to solid matrices. At Toyota, iron particles approximately 100 $\mu \mathrm{m}$ in size were dispersed in a silicone rubber and aligned before the curing [11]. Lord Co. also used silicone rubber as the matrix and aligned their particles before the curing $[17,18]$. One difference, compared to the materials produced by Toyota, was that the particles were carbonyl iron (as in MR fluids), which are spherical with a diameter of 1$10 \mu \mathrm{m}$. Ford also used carbonyl iron particles and they aligned the particles before the vulcanization, but they used natural rubber as their matrix $[19,20]$.

\subsection{Particles}

The particles of choice, for both MR fluids and MR solids, are iron particles. This is because of their high permeability, low remnant magnetisation and high saturation magnetisation [5]. High permeability and high saturation magnetisation provide high interparticle attraction, and thereby a high MR effect [21]. Alloys of iron and cobalt or nickel which have an even larger saturation magnetisation than iron also have been used in MR fluids [22]. Nevertheless, the use of these particles in MR materials is not as widespread as the use of iron. Remnant particles will still stick together when the magnetic field is turned off, and will not therefore show a completely reversible MR effect. Furthermore, the sedimentation stability and redispersibility of remnant particles in a fluid will be less, due to the permanent forces between the particles [23]. The particles should be large enough to support several magnetic domains, otherwise the MR effect will be much smaller, or none at all [21]. In fact, fluids with nano-sized particles are referred to as "ferrofluids" or "magnetic fluids", and they react in a completely different way in a magnetic field. If a magnetic field is applied through a ferrofluid, the entire fluid will move to the strongest field, due to the Brownian motions of the particles which hinders them to approach each other [24]. An MR effect can be measured if the magnetic field is completely homogeneous, but it is much smaller than for MR fluids [21]. The dependence of the yield stress of fluids on particle size is very large up to $1 \mu \mathrm{m}$ in diameter [25]. However, even for particles with diameters of several micrometers, the yield stress of MR fluids increases with increasing particle size [26]. Carbonyl iron, spherical iron particles with a diameter of some micrometers, is the particle type that has been most frequently used in MR materials [5]. 


\section{Experimental}

\subsection{Materials}

The iron particles used were of two different types: irregularly shaped pure iron particles from Höganäs AB, Höganäs, Sweden, and spherical carbonyl iron powder (CI). Both types are pure iron particles $(>99 \%)$, but they differ from each other in shape and size. The particles from Höganäs were:

ASC300 (particle size $<60 \mu \mathrm{m})$

ASC100.29 (particle size $<180 \mu \mathrm{m}$ )

AT40.29 (particle size $\approx 200 \mu \mathrm{m}$ ).

The particle size distributions of these powders are shown in Figure 2.1. The carbonyl iron was SQ from BASF A.G., Ludwigshafen, Germany, with a particle size of 3.9 $5.0 \mu \mathrm{m}$.

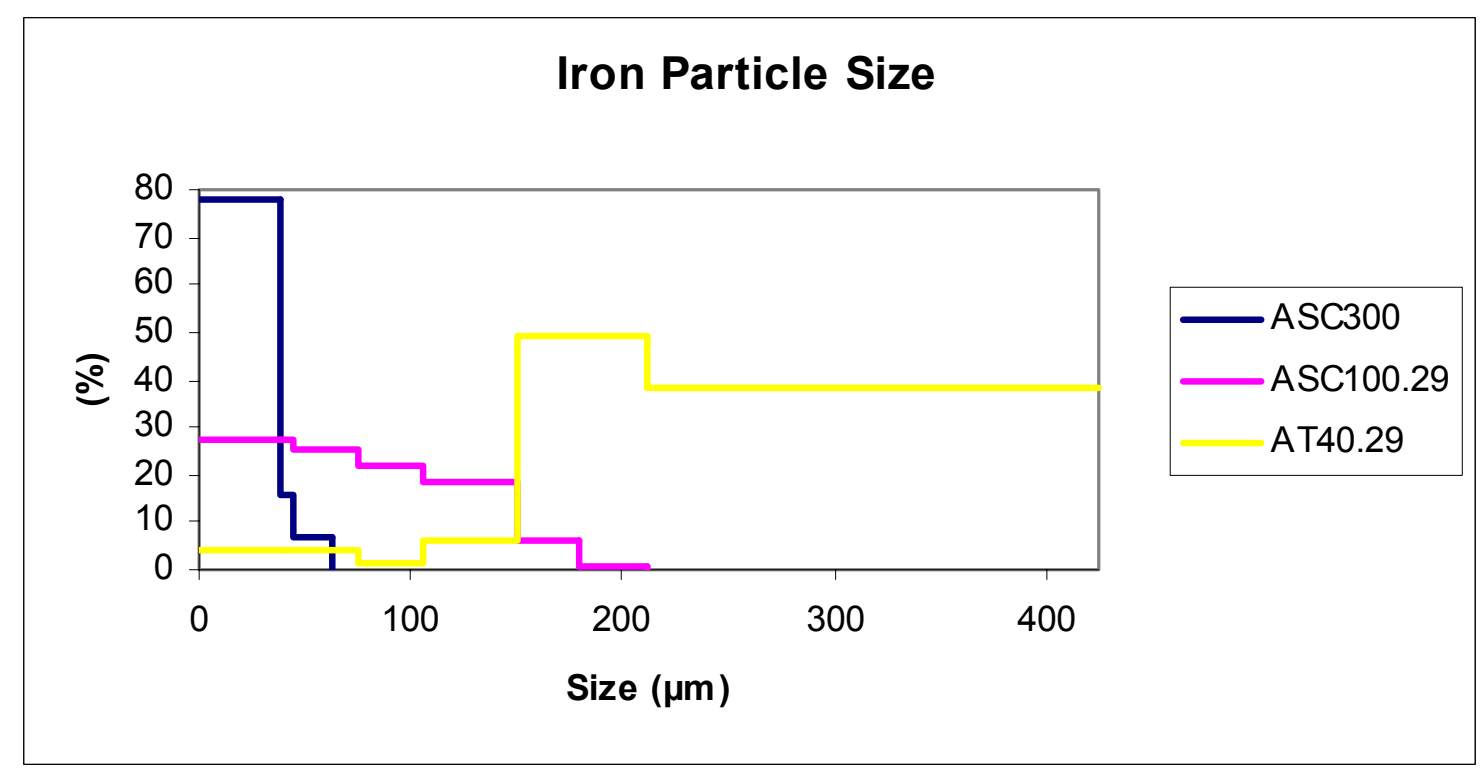

Figure 2.1. Particle size distributions of the Höganäs iron powders.

The matrix materials used were four nitrile rubbers with different acrylonitrile (ACN) contents:

Perbunan 1845 (18\% ACN)

Perbunan $3445(34 \%$ ACN)

Krynac 45.50 (45\% ACN)

Krynac 50.75 (50\% ACN)

Natural rubber (SMR GP), and a mix of polybutadiene (Buna CB55) and natural rubber were used as materials with $0 \% \mathrm{ACN}$. All the matrix materials used, except for the 
natural rubber, were made by Bayer A.G., Leverkusen, Germany. The vulcanisation system used was a conventional sulphur curing system. The recipe was in all cases: 100 phr rubber, $5 \mathrm{phr}$ zinc oxide $(\mathrm{ZnO}), 1 \mathrm{phr}$ stearine, $1.5 \mathrm{phr}$ sulphur, and $1.5 \mathrm{phr}$ MBTS. In some cases, the material was plasticised, the plasticisers being di-2-ethylhexylphthalate (DEHP) in the nitrile rubbers, and hydrocarbon oil, Nytex 840 in natural rubber. The maximum plasticiser content was $17 \mathrm{phr}$, in both the nitrile and the natural rubber. The iron particles were mixed into the rubber together with the vulcanisation system in a Brabender mixer. All the materials were vulcanised at $150^{\circ} \mathrm{C}$ for 30 minutes under a pressure of approximately $12 \mathrm{MPa}$.

\subsection{Methods}

\subsubsection{MR measurements}

The magnetorheological effect was evaluated by measuring the dynamic shear modulus with and without an applied magnetic field. This was done on a double lap shear specimen, using an Instron 8032 dynamic testing machine, equipped with an electromagnet (Figure 2.2). The rubber segments were approximately $20 \times 15 \times 2 \mathrm{~mm}$, and they were sandwiched between brass or steel plates. The rubber was fixed to the plates by a cyanoacrylate adhesive. The schematic equipment set-up is shown in Figure 2.3.

When brass plates were used as fixtures for the samples, the modulus was measured at frequencies from 1 to $21 \mathrm{~Hz}$. The peak-to-peak strain amplitude was $5 \%$ at $1 \mathrm{~Hz}$ and decreased with increasing frequency down to $1.2 \%$ at $21 \mathrm{~Hz}$. When a current of $20 \mathrm{~A}$ was used, the electromagnet was capable of applying a magnetic field of $170 \mathrm{kA} / \mathrm{m}$ through the samples between the brass plates. The resulting magnetic induction through samples containing $28 \%$ by volume of iron particles was then $0.24 \mathrm{~T}$.

In the measurements with steel plate fixtures, the peak-to-peak strain amplitude was constant, $2 \%$, throughout the measurement. The currents used through the coil of the magnet were 5, 2, and $1 \mathrm{~A}$. These currents generated magnetic fields of 525, 280, and 130 $\mathrm{A} / \mathrm{m}$ respectively, which resulted in magnetic inductions of about $0.8,0.55$, and $0.3 \mathrm{~T}$ through samples with approximately $32 \%$ by volume of iron. The magnetic fields were measured by a FH31Gaussmeter from Magnet-Physik Dr. Steingroever GmbH, Germany, and were approximately constant over the surfaces of the samples.

The modulus was first measured as a frequency sweep without any magnetic field. The measurement was then repeated with the applied magnetic field. This procedure was then repeated. Two sets of samples of each material were evaluated in this way.

To determine the amplitude dependence of the MR effect, the modulus was measured at frequencies of 2, 10, and $50 \mathrm{~Hz}$, and strain amplitudes of 1.1, 2.8, 5.4, and $11 \%$. The modulus was measured with and without an applied magnetic field. The current through the coil during the tests with magnetic field was 5A, and the steel fixtures were used, resulting in a total magnetic induction of about $0.8 \mathrm{~T}$. 


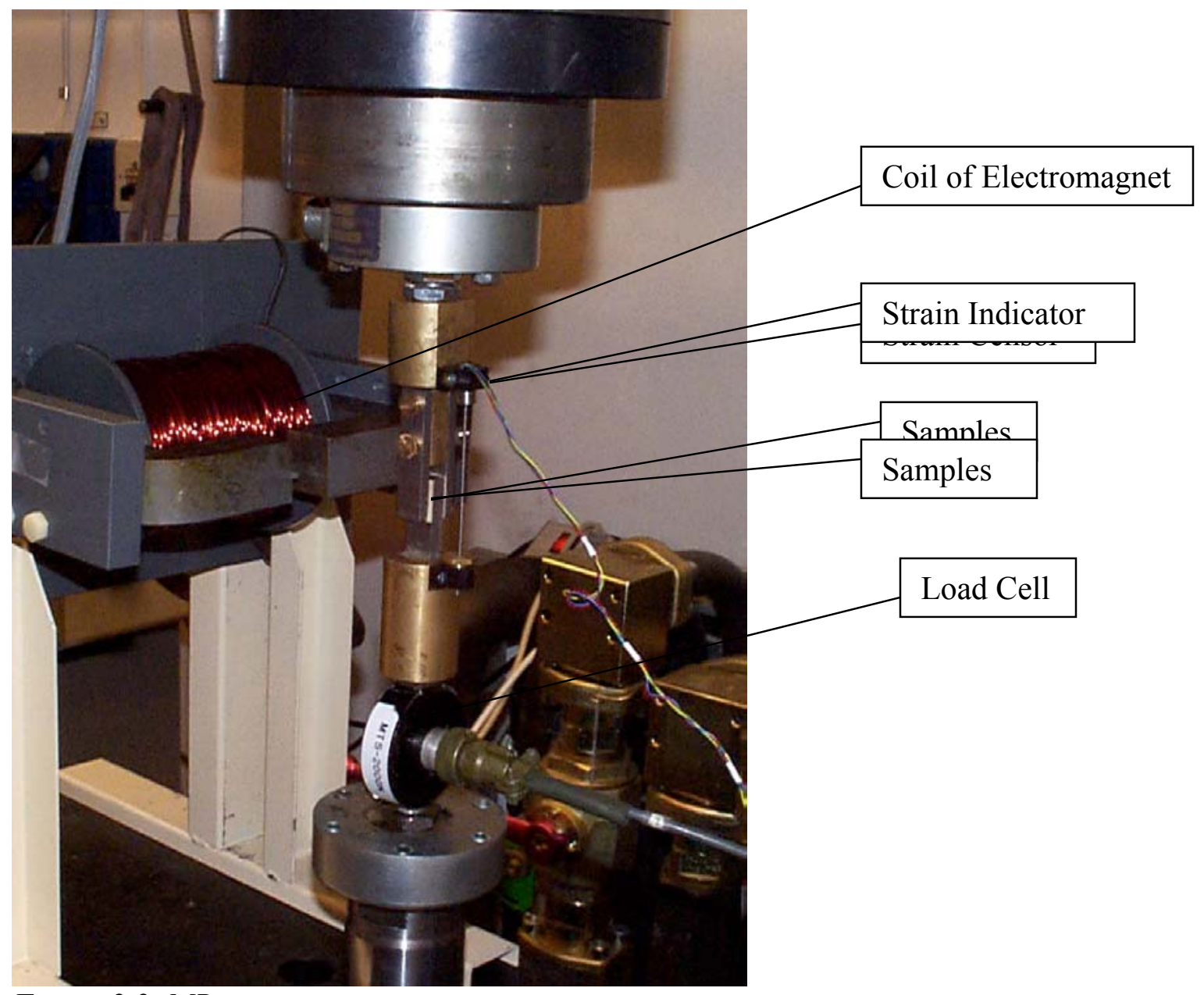

Figure 2.2. MR measurement equipment

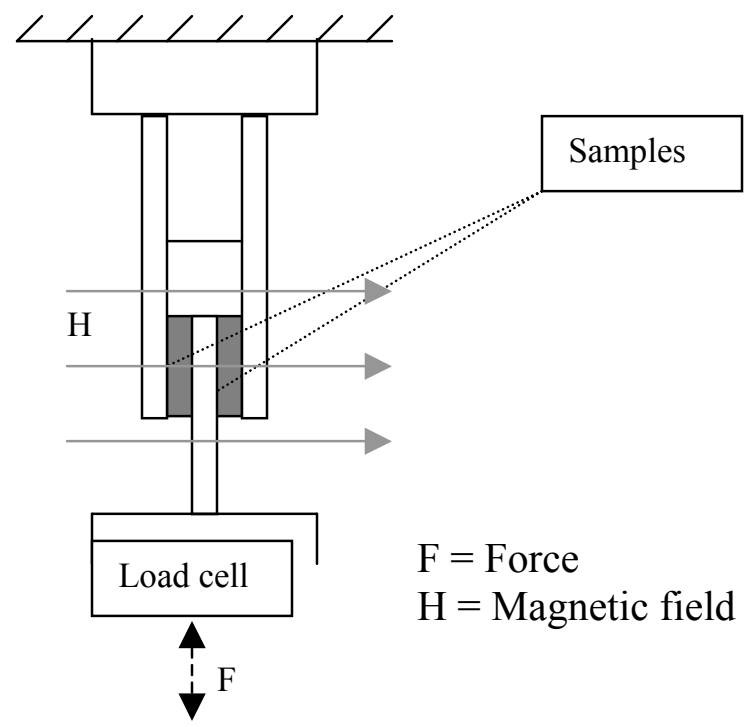


Figure 2.3. MR measurement equipment (Schematic)

\subsubsection{High frequency MR measurements}

The MR measurements in the audible frequency range were performed using a specially designed test rig (Figure 2.4). The samples were of the same size and shape as in the MR measurements at lower frequencies, and the fixtures with steel plates were used. The fixtures with the samples were mounted between an electro-dynamic vibration generator and a motion-reducing blocking mass. The test set-up was decoupled from the base by three cylindrical, auxiliary vibration isolators, manufactured by Trelleborg AVS, with a hardness of 40 IRHD. The vibration generator, which was resiliently suspended from the roof by rubber springs, excited the force distribution plate of the sample fixture, and the motion was measured by two piezo-electric accelerometers on the force distribution plate and one on the bottom of the blocking mass. The acceleration multiplied by the mass of the blocking mass is the blocking force required in the assessment of the dynamic shear modulus.

The tests were carried out at room temperature, $21.0 \pm 0.5^{\circ} \mathrm{C}$, within a frequency domain of 100 to $1250 \mathrm{~Hz}$. To increase the signal-to-noise ratio, the excitation signal was a stepped sine wave signal; starting from $100 \mathrm{~Hz}$, with a constant frequency increment of $10 \mathrm{~Hz}$. The signal was recorded within a $10 \mathrm{~Hz}$ bandwidth, averaged 5 times and delayed $1000 \mathrm{~ms}$ between each recording. The total mass of the blocking mass was approximately $210 \mathrm{~kg}$.

\subsubsection{Density measurements}

In order to confirm the iron particle content of the samples, the density of each sample was determined before the MR measurements. The density was calculated applying the Archimedes principle according to:

$$
\text { Density }=\frac{\text { Mass in air }}{\text { Buoyancy in ethanol }} \times \text { Density of ethanol }
$$

Density of ethanol $=0.79 \mathrm{~g} \mathrm{~cm}^{-3}$.

A Mettler Toledo AE100 balance and a Mettler density determination kit 33360 were used for the measurements. The volume percentage of iron particles was calculated according to:

$$
\mathrm{c}_{\text {iron }}=\frac{\rho_{\text {tot }}-\rho_{\text {rubber }}}{\rho_{\text {iron }}-\rho_{\text {rubber }}}
$$


where $\rho_{\text {tot }}$ is the density of the whole material, $\rho_{\text {rubber }}$ is the density of the matrix material, and $\rho_{\text {iron }}$ is the density of iron $=7.9 \mathrm{~g} \mathrm{~cm}^{-3}$.

\subsubsection{Tensile test}

The stress-strain properties of the materials were evaluated using an Instron 5566 tensile testing machine. The samples were "dog bones" with a cross-section of approximately $2 \times 3 \mathrm{~mm}$. The crosshead speed was $500 \mathrm{~mm} / \mathrm{min}$. Because of the varying degrees of slippage of the samples at high strains, the stress at break was considered to be a more appropriate property for comparison than the strain at break.

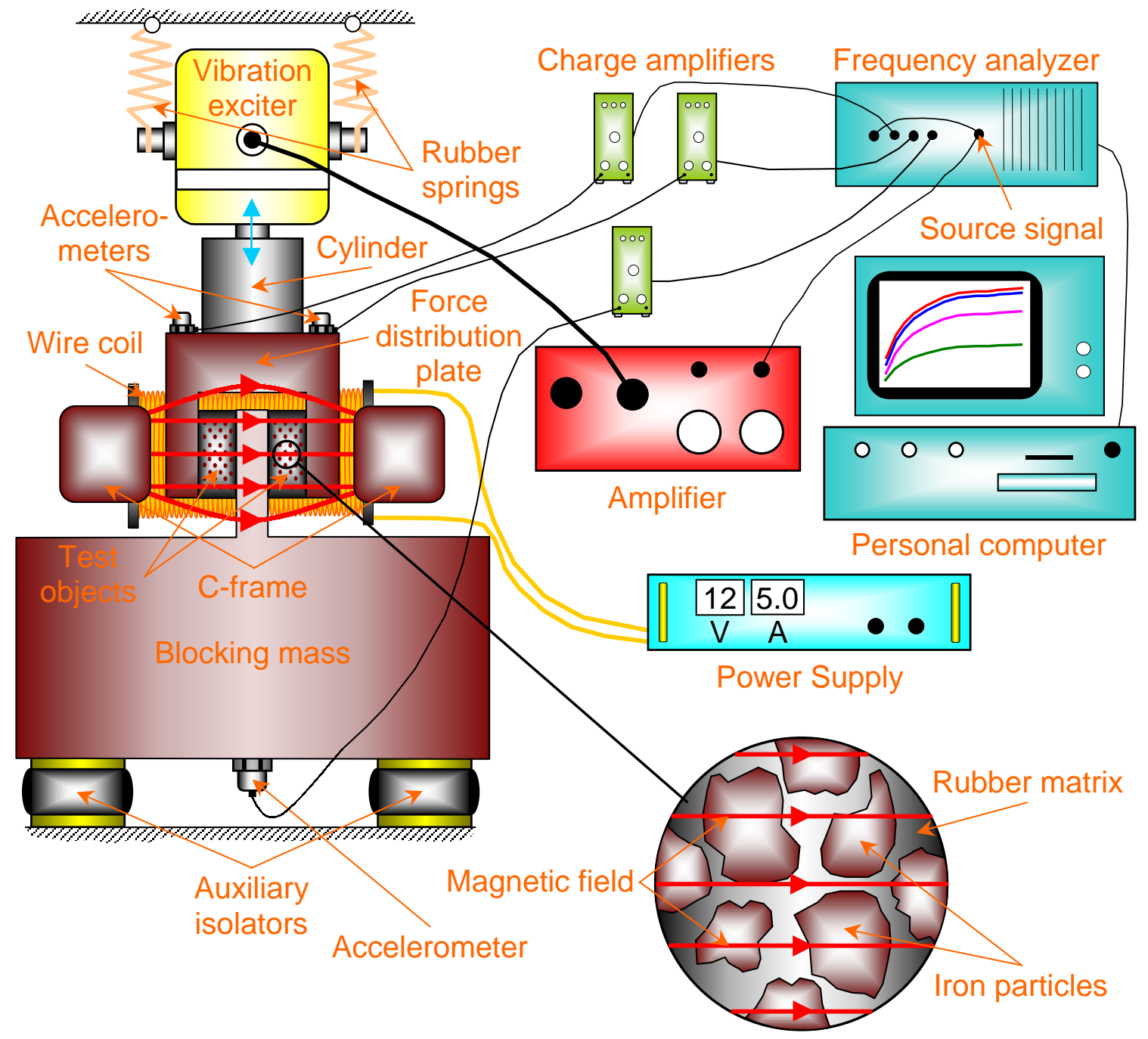

Figure 2.4. High frequency MR measurement equipment

\subsubsection{Rheometer measurements}

The viscosity of the uncured rubber materials with zinc oxide and stearine was measured using a stress-controlled "Stresstech melt HR" rheometer from Rheologica. The oscillation frequency was $1 \mathrm{~Hz}$ and the stress was increased from $0.1 \mathrm{kPa}$ to $10 \mathrm{kPa}$ 
during the measurements. The rheometer was also used to measure the modulus of the cured rubber materials without iron particles, in order to evaluate the influence of the adhesive on the modulus values from the MR measurements. In these modulus measurements, the frequency was 1 to $21 \mathrm{~Hz}$, as in the MR measurements.

\section{Results and discussion}

\subsection{Validity of the MR measurements}

Figure 3.1 shows the modulus of the pure nitrile rubbers as measured by the "MR equipment" and by the rheometer. Absolute values of the modulus are very dependent on the measurement method used, which means that is difficult to compare modulus values obtained by different methods. Nevertheless, the values of the modulus obtained from the MR measurements are remarkably higher than those from the rheometer tests. The adhesive used in the MR tests probably influences the values, so that the measured values are somewhat higher than the real values. This means that the actual relative MR effect is larger than reported, since the adhesive will influence the measurements with and without a magnetic field in the same way, so that the reported absolute MR effect is correct.

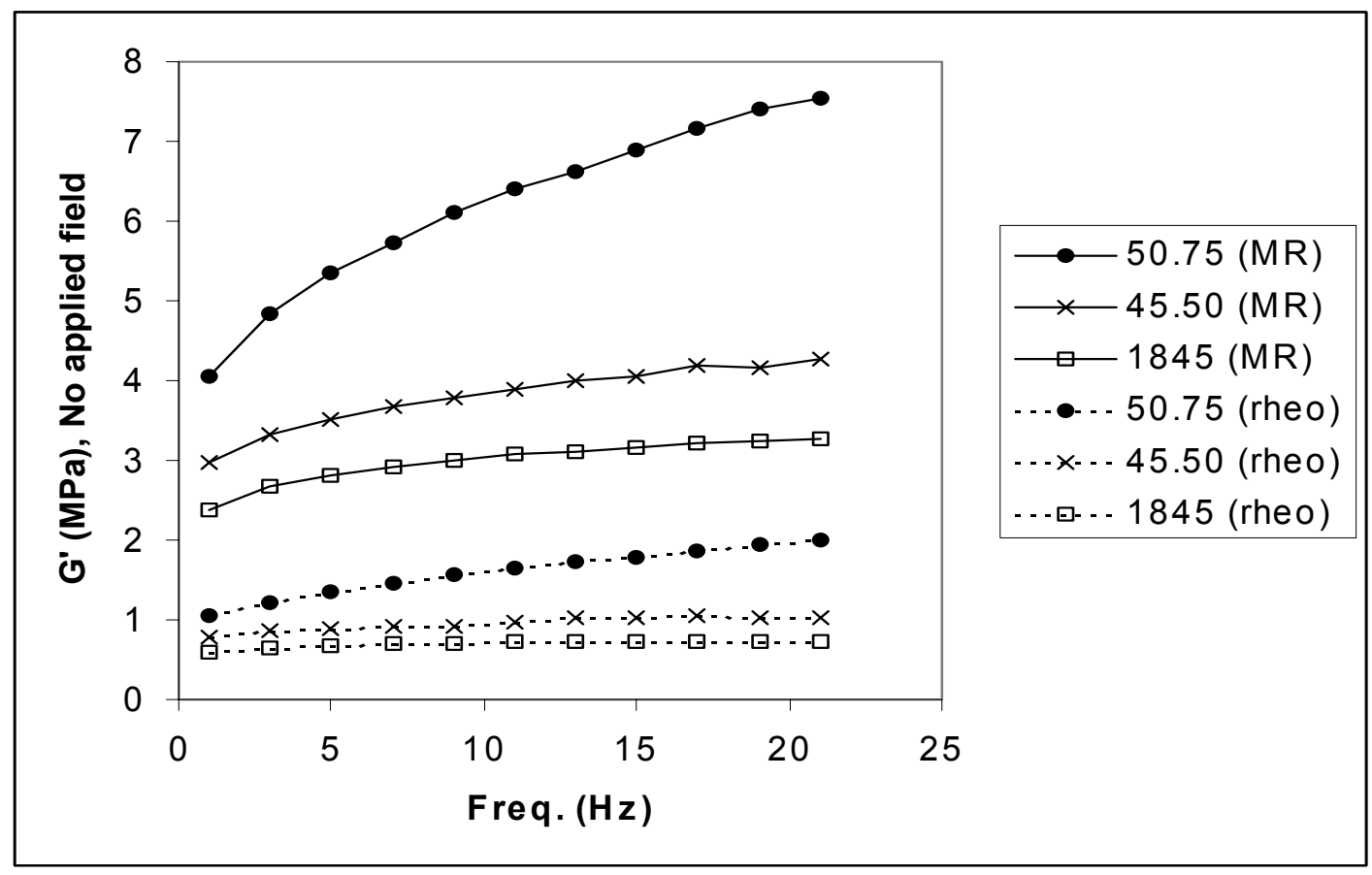

Figure 3.1. Zero-field modulus (mean value of two samples) of rubbers without iron particles as measured by the MR equipment (MR) and with a rheometer (rheo).

Figure 3.2 shows the increase in shear modulus due to the magnetic field, for pure Perbunan 1845 without iron, as measured using the brass plates and the steel plates. The influence of the magnetic field on the rubber is close to zero, indicating that the measurement method is valid, i. e. the increase in modulus seen when a magnetic field is applied is caused by the material, and not by the equipment. 


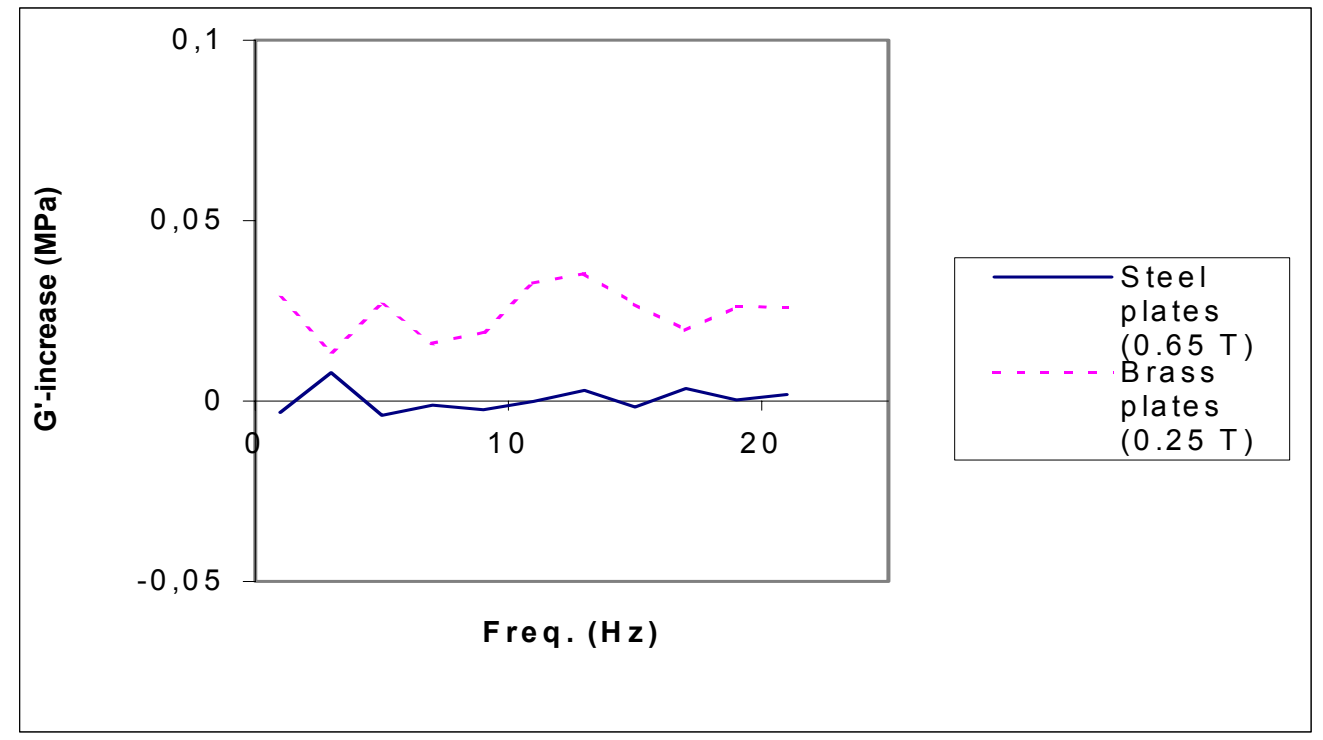

Figure 3.2 Absence of MR effect in unfilled Perbunan 1845

The relative MR effect of Perbunan 3445 is shown in Figure 3.3 as a function of the iron content. The results of measurements with steel plate fixtures are compared with results obtained with the brass plate fixtures. The frequency chosen for the comparison is $11 \mathrm{~Hz}$, because at $11 \mathrm{~Hz}$ the strain amplitude of the measurements with brass plates was $2 \%$, as in the measurements with steel plates. There was good agreement between the measurements when the magnetic induction was similar, about $0.3 \mathrm{~T}$. However, the scatter was much smaller in the measurements with steel plates. The change of material in the fixtures, from brass to magnetic steel, does not influence the results of the measurements, but the steel fixtures permit measurements to be made with stronger magnetic fields than the brass plates.

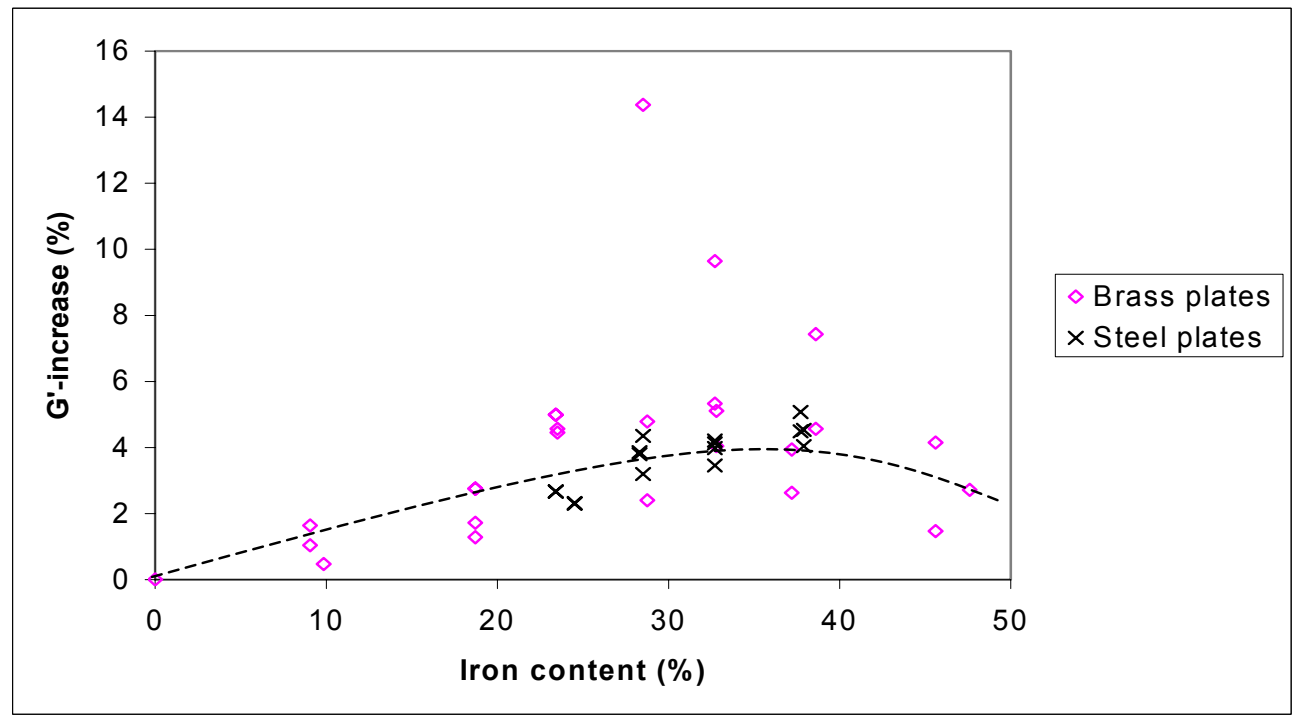


Figure 3.3.Relative MR effect as a function of iron content for Perbunan 3445 with ASC300, as measured with brass plates and with steel plates, and with a magnetic field of about $0.3 T$.

\subsection{Critical Particle Volume Concentration (CPVC)}

The magnetorheological effect in Perbunan 3445 with different contents of ASC300 and carbonyl iron, measured at $7 \mathrm{~Hz}$ with the brass plates, is shown in Figure 3.4. Figure 3.4a shows the absolute increase (in MPa) in shear modulus associated with an applied magnetic field and Figure 3.4b shows the relative effect (in \%). As can be seen, the effect was significantly larger in the materials with ASC300 than in the materials with carbonyl iron $(\mathrm{CI})$. There a maximum in the relative magnetorheological effect at a content of about $30 \%$ by volume of ASC 300 . At higher concentrations, the stiffening of the material, caused by increase in the content of filler, was larger than the increase in MR effect.

Figure 3.5 shows the stress at break as a function of iron content. It is almost constant in the iron concentration range up to approximately $30 \%$ by volume. At higher concentrations, the mechanical properties deteriorate rapidly with increasing amounts of iron.

The MR effect of 3445 with approximately $28 \%$ by volume of the different iron powders, as measured using the brass plates, is shown in Figure 3.6. With the particles from Höganäs the difference in the MR effect is almost insignificant. However, the effect appears to be slightly higher in materials with ASC300 than in materials with even larger particles.

Figure 3.7 shows the MR effect of different matrix materials with approximately $28 \%$ of ASC300 or carbonyl iron, as measured using the brass plates. As can be seen, the matrix material does not influence the MR effect when ASC300 is used. With carbonyl iron, on the other hand, the MR effect is greater in the materials with hard matrix materials than in the materials with softer matrix. In fact, the MR effect in hard matrix materials (Krynac 45.50 and Krynac 50.75) with carbonyl iron is similar to that in materials with ASC300. 

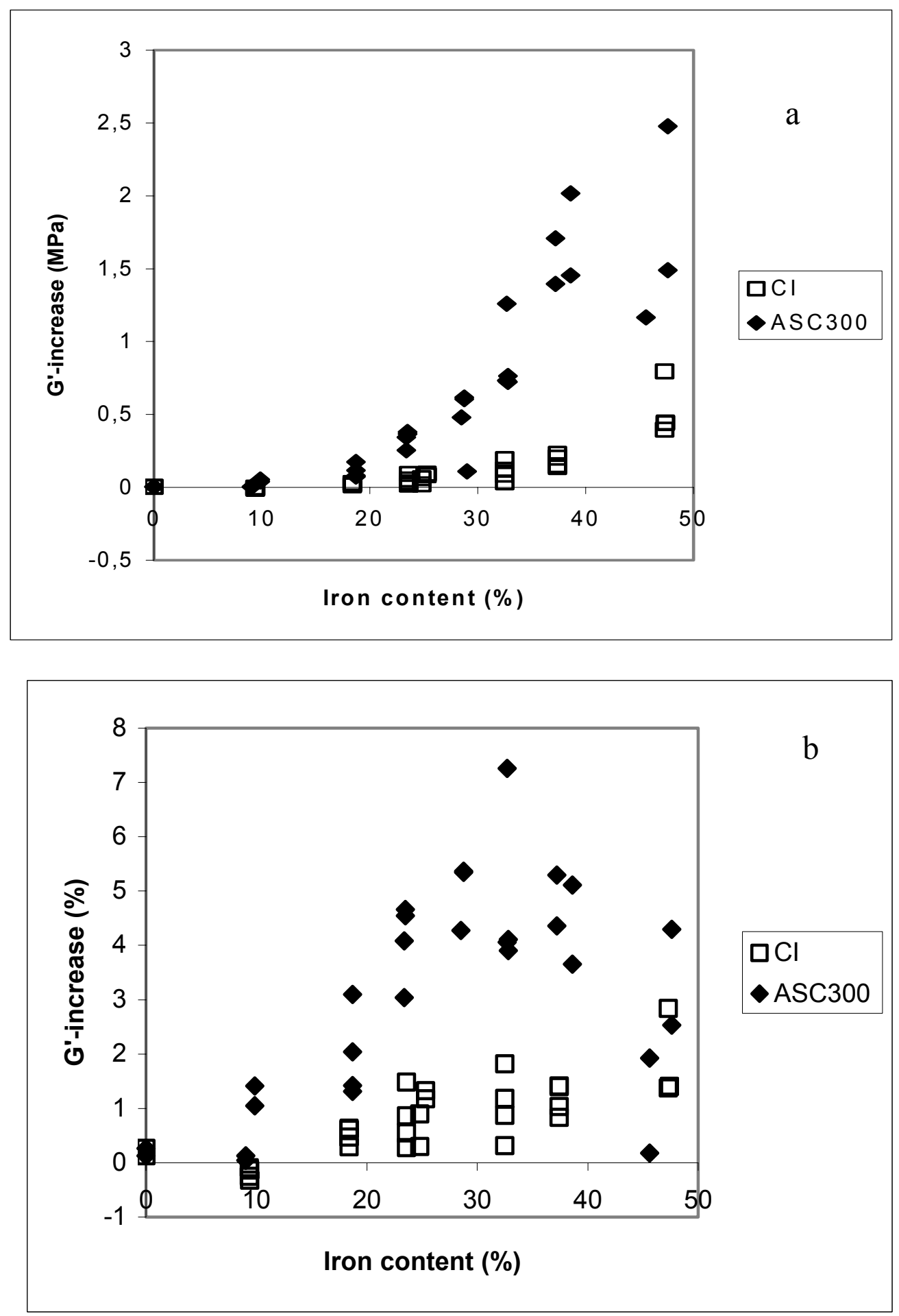

Figure 3.4. The magnetorheological effect of Perbunan 3445 with ASC300 and carbonyl iron (CI), displayed in 3.4a as the absolute increase in shear modulus (in MPa), and in $3.4 b$ as the relative increase in shear modulus (in \%). 


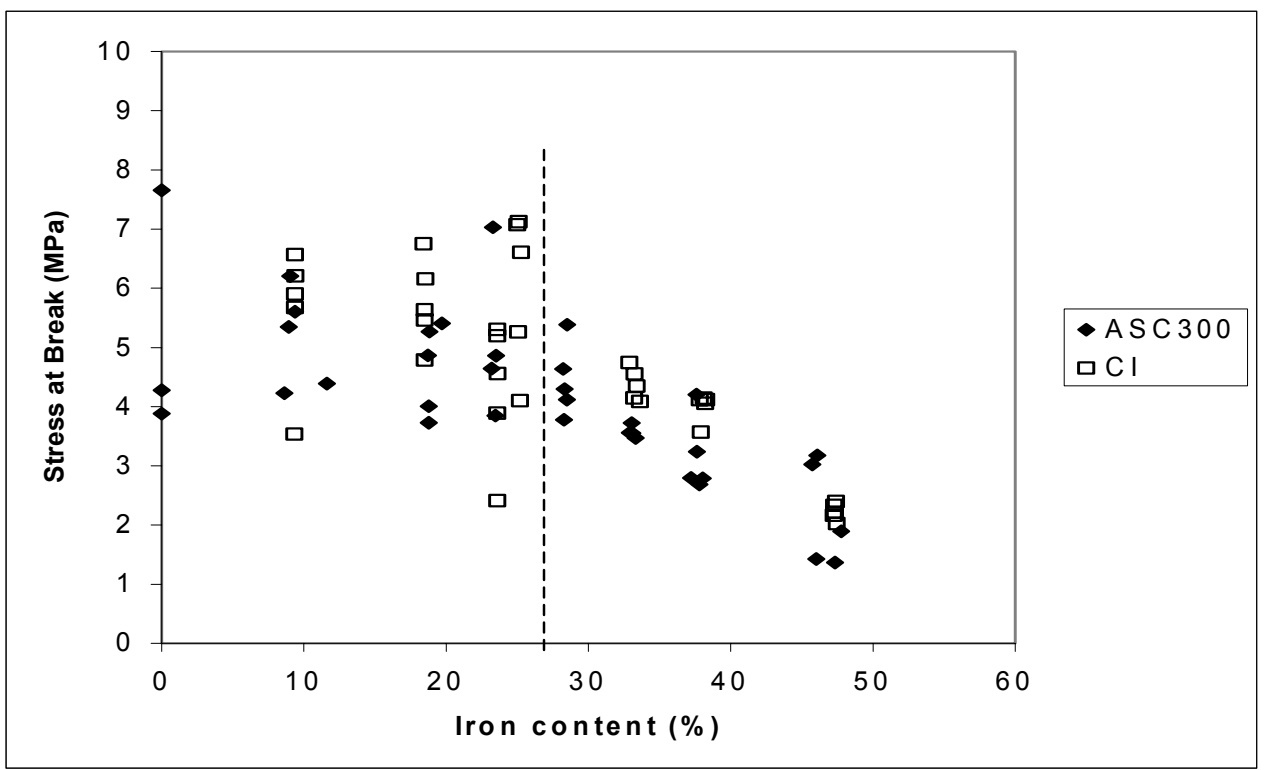

Figure 3.5 Stress at break of Perbunan 3445 with ASC300 and carbonyl iron (CI).

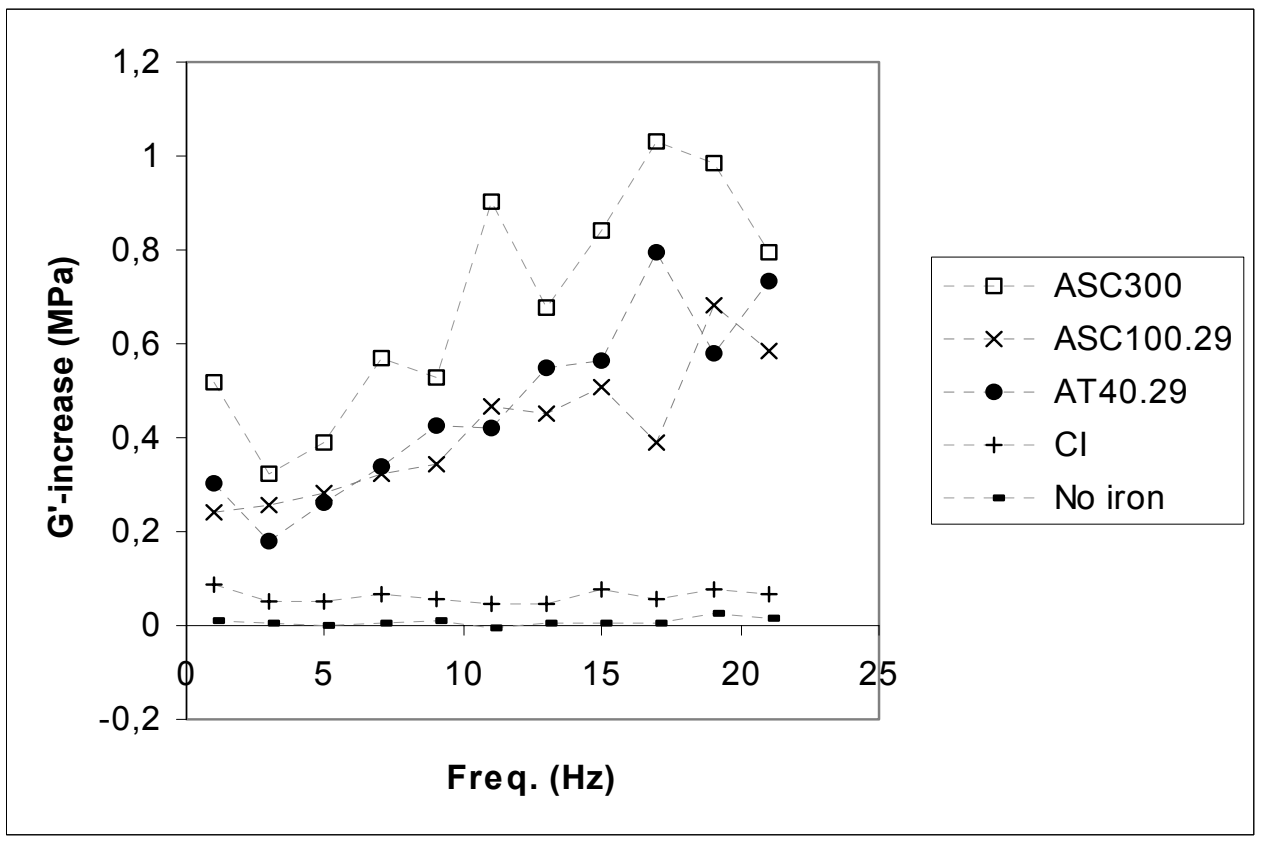

Figure 3.6 MR effect in Perbunan 3445 with approximately 28\% of different iron particles, and the absence of MR effect in unfilled Perbunan 3445. 

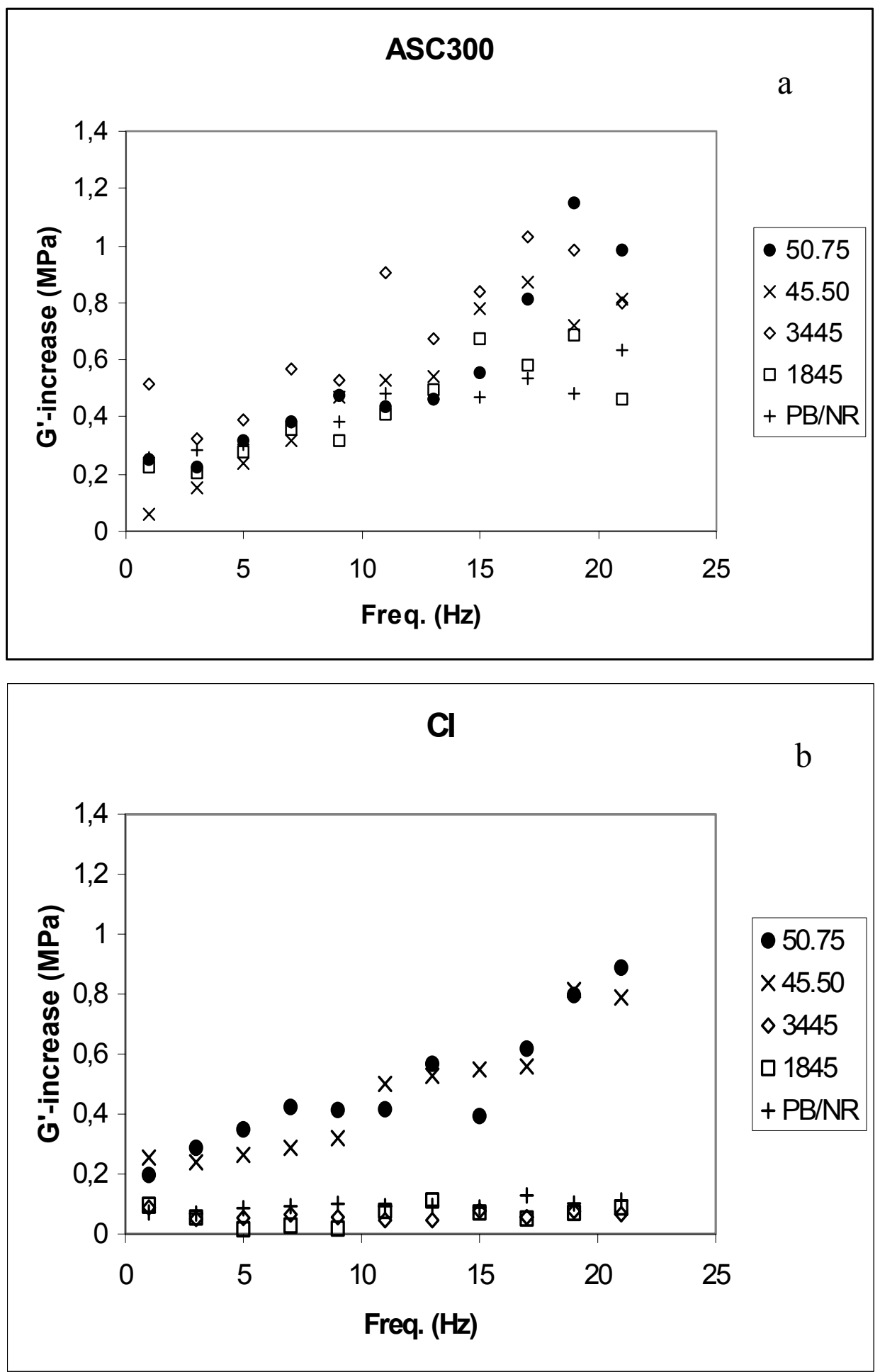

Figure 3.7. Absolute MR effect of different matrix materials with 28 vol\% of (a) ASC300 and (b) carbonyl iron. 
The MR effect was greater than first expected in the materials with the large irregularly shaped particles, considering the low magnetic field in these measurements and the fact that the particles are not aligned within the matrix. Magnetic forces are strongly dependent on the distance. If the distance between the particles is $r$, the interaction energy between two magnetic dipoles is inversely proportional to $r^{3}[17,18]$. When the particles within MR solids have been aligned by magnetic fields, the distance between the particles has been minimised in the direction of the magnetic field, and this leads to a substantially larger MR effect than is obtained in materials with unaligned carbonyl iron. Furthermore, alignment of the particles decreases the zero-field shear modulus [27]. The maximum increase in shear modulus of these materials has been reported to be approximately 0.7 $\mathrm{MPa}$ at magnetic fields of about $0.8 \mathrm{~T}$ [17-19]. Even though it is difficult to compare results obtained in different equipment, it is interesting to note that isotropic MR rubber materials with irregularly shaped particles show an increase in modulus of about $0.4 \mathrm{MPa}$ at $10 \mathrm{~Hz}$ and $0.24 \mathrm{~T}$ (Figure 3.7a), whereas rubber materials with aligned carbonyl iron show an increase of $0.7 \mathrm{MPa}$ at $10 \mathrm{~Hz}$ and $0.85 \mathrm{~T}$ [18].

The large MR effects apparent in some of our unaligned materials are probably due to the fact that the distances between the particles are quite small, even though the particles are not aligned. When iron particles are stored in a container, there are large amounts of air between the particles. This means that the "apparent density" of the powder is much less than the density of solid iron. If the air is replaced by rubber, the result is a rubber filled with a critical amount of iron. This "Critical Particle Volume Concentration" (CPVC) is the concentration at which the particles are in physical contact with each other and the voids between them are completely filled with rubber. At lower concentrations, the particles are separated by more rubber, and at higher particle concentrations, the amount of rubber is insufficient to fill all the voids between the particles. This means that at concentrations higher than the CPVC, the mechanical properties, such as stress at break, will deteriorate rapidly, and the stiffening of the material due to the increase in filler content will be large. It also means that the MR effect will not increase much with increasing particle content above the $\mathrm{CPVC}$, and that the relative $\mathrm{MR}$ effect will show a maximum around the CPVC.

The CPVC for the different powders can be calculated from their apparent densities. The apparent density of ASC 300 is $2.88 \mathrm{~g} \mathrm{~cm}^{-3}$ (Höganäs specifications). The volume concentration of iron in a container with ASC300 is then:

$\mathrm{c}_{\text {iron }}=\frac{100 \times \rho_{\text {app }}}{\rho_{\text {iron }}}=\frac{100 \times 2.88}{7.9} \approx 36.5 \mathrm{vol} \%$

This means that the CPVC for MR rubber materials with this iron powder is $36.5 \%$ by volume. Similarly the CPVC for ASC100.29 is $38.2 \%$ by volume, and for AT40.29, $38.7 \%$ by volume. 
This approach explains several of the features in our results. The maximum MR effect in the materials with ASC300 is obtained at a particle concentartion of about $30 \%$ by volume, which is is close to the CPVC of this powder.

At about the same concentration, the mechanical properties start to deteriorate more rapidly with increasing iron content. The shift towards lower concentrations can be explained by the fact that the dispersion of the particles is not perfect, or by the large scatter in the results from the measurements with brass plates. In the measurements with steel plates, the maximum in MR effect is closer to the calculated value of the CPVC of ASC 300. The small differences in MR effect among materials with the same amounts of ASC300, ASC100.29, and AT40.29 are due to the small differences in CPVC of these powders.

With carbonyl iron, the situation is more complicated due to the tendency of these particles to form aggregates. The aggregates may look quite similar to the larger irregularly shaped particles and the apparent density of the powder may be about the same. Nevertheless, the particles can be packed more closely together if the aggregates are destroyed. Therefore, the CPVC for materials with carbonyl iron may be larger than the values calculated from the apparent density of the powder suggest, if the particles are well dispersed in the matrix. The larger MR effect in carbonyl iron containing materials in hard matrices than in materials with the same amount of carbonyl iron in softer matrices is probably due to the fact that the particles are less well dispersed within the matrix when the viscosity is higher. Aggregates, which act like larger irregular particles, are thus present to a greater extent.

\subsection{Increasing the MR Effect}

The absolute MR effect, as measured using the steel plates, as a function of the applied magnetic field is shown in Figure 3.8 for different iron particle concentrations. As can be seen, the MR effect does not increase as much with increasing field strength above 280 $\mathrm{kA} / \mathrm{m}$, as at lower field strengths. This suggests that the materials start to become magnetically saturated at this magnetic field level. The behaviour is similar to that observed with anisotropic MR rubbers [19]. However, the absolute MR effect in the isotropic materials is much greater than the largest absolute MR effect in previously reported anisotropic materials [20], also at higher magnetic fields. This can perhaps to some extent be explained by the smaller strain amplitudes used in our measurements. Figure 3.9 shows the MR effect as a function of the strain amplitude, and it is evident that the MR effect decreases rapidly with increasing applied strain within the measured strain range, but that it is independent of the frequency. Nevertheless, if the results in figure 3.9 are compared with the results of Nichols et al [20] obtained under similar conditions, the MR effect is still approximately twice as large for the isotropic MR rubber as for the materials with aligned carbonyl iron (0.8 MPa at $0.8 \mathrm{~T}$ and $5.4 \%$ strain, compared to 0.4 $\mathrm{MPa}$ at $1 \mathrm{~T}$ and $5.5 \%$ strain). 




Figure 3.8. Absolute MR effect as a function of the applied magnetic field for Perbunan 3445 with different iron concentrations

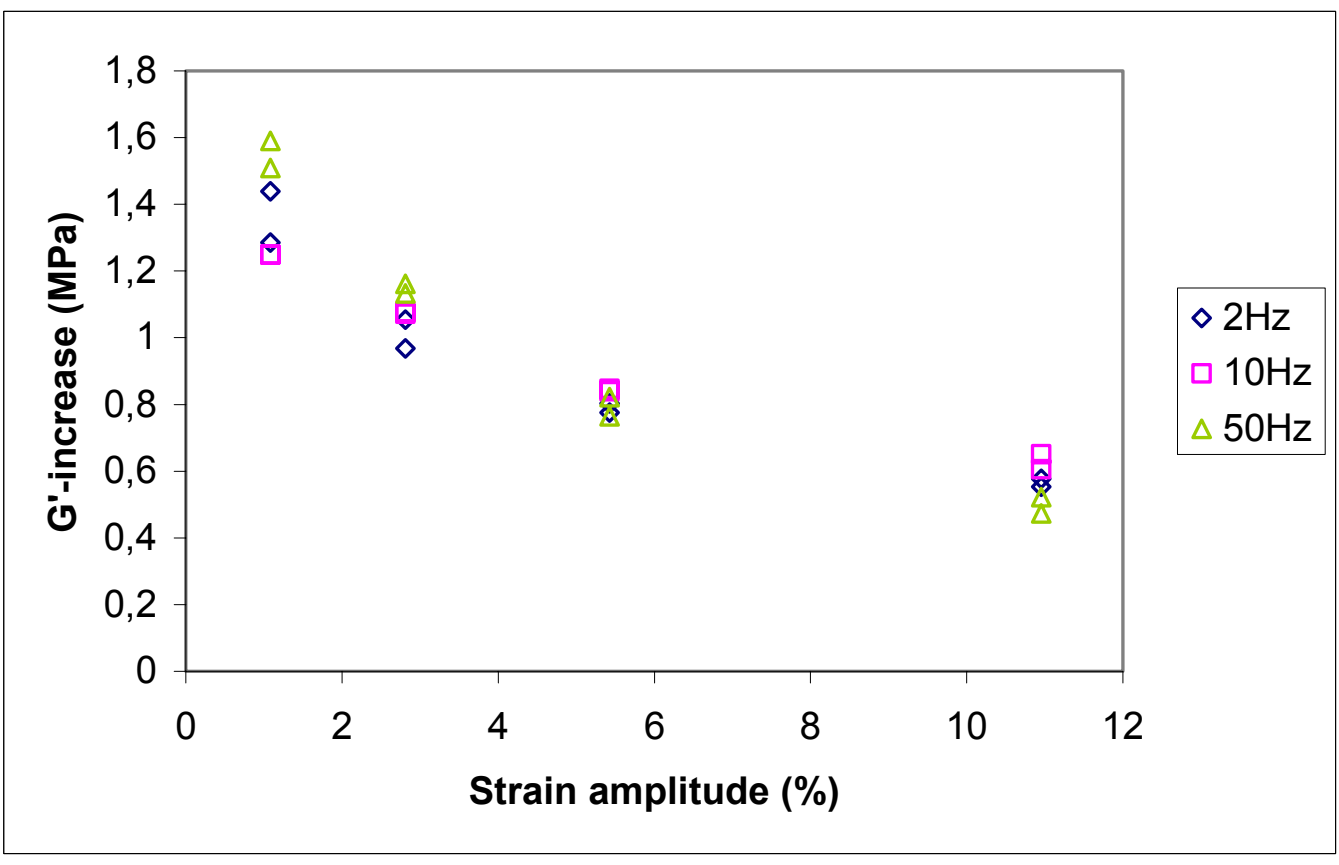

Figure 3.9. Absolute MR effect of Perbunan 1845 with $32 \%$ by volume of ASC300, measured at different strain amplitudes and frequencies. 
Figure 3.10a shows that the absolute MR effect in isotropic MR rubbers increases exponentially with increasing iron concentration below the CPVC, regardless of the matrix material. Deviations from this behaviour, such as the slightly lower absolute MR effect in the materials with NR matrices, may be explained by local anisotropy in the samples, and differences in the degree of dispersion. Even when the term "isotropic" is used, the materials always have regions of local anisotropy. This local anisotropy may be beneficial for the MR effect if it is in the direction of the field, but it may decrease the MR effect if it is perpendicular to the field. Aggregation of particles will decrease the $\mathrm{CPVC}$, the iron concentration where the MR effect is optimal. One way to determine the actual CPVC for the materials is to use electric techniques, such as volume resistivity measurements. When the concentration is high enough for the particles to form a continuous network throughout the material, i.e. at the CPVC, the electrical properties change rapidly with increasing concentration, and this makes such methods suitable for determining the actual CPVC for any matrix/iron particle combination [16].

The fact that the absolute MR effect is independent of the matrix material, means that softer matrix materials will increase the relative MR effect, as can be seen in figure $3.10 \mathrm{~b}$, where the addition of plasticisers to the materials has increased the relative MR effect substantially.

These results show that, in order to achieve a large MR effect, the iron particle concentration has to be very close to the CPVC, otherwise the material becomes saturated at too low magnetic fields. It is not necessary to apply very large magnetic fields, since the MR effect does not increase much with increasing magnetic field strength when materials have started to saturate magnetically. The MR effect at about $0.8 \mathrm{~T}$ is only slightly larger than that at $0.5 \mathrm{~T}$. A simple way to increase the relative MR effect, is to plastisicer the matrix. 

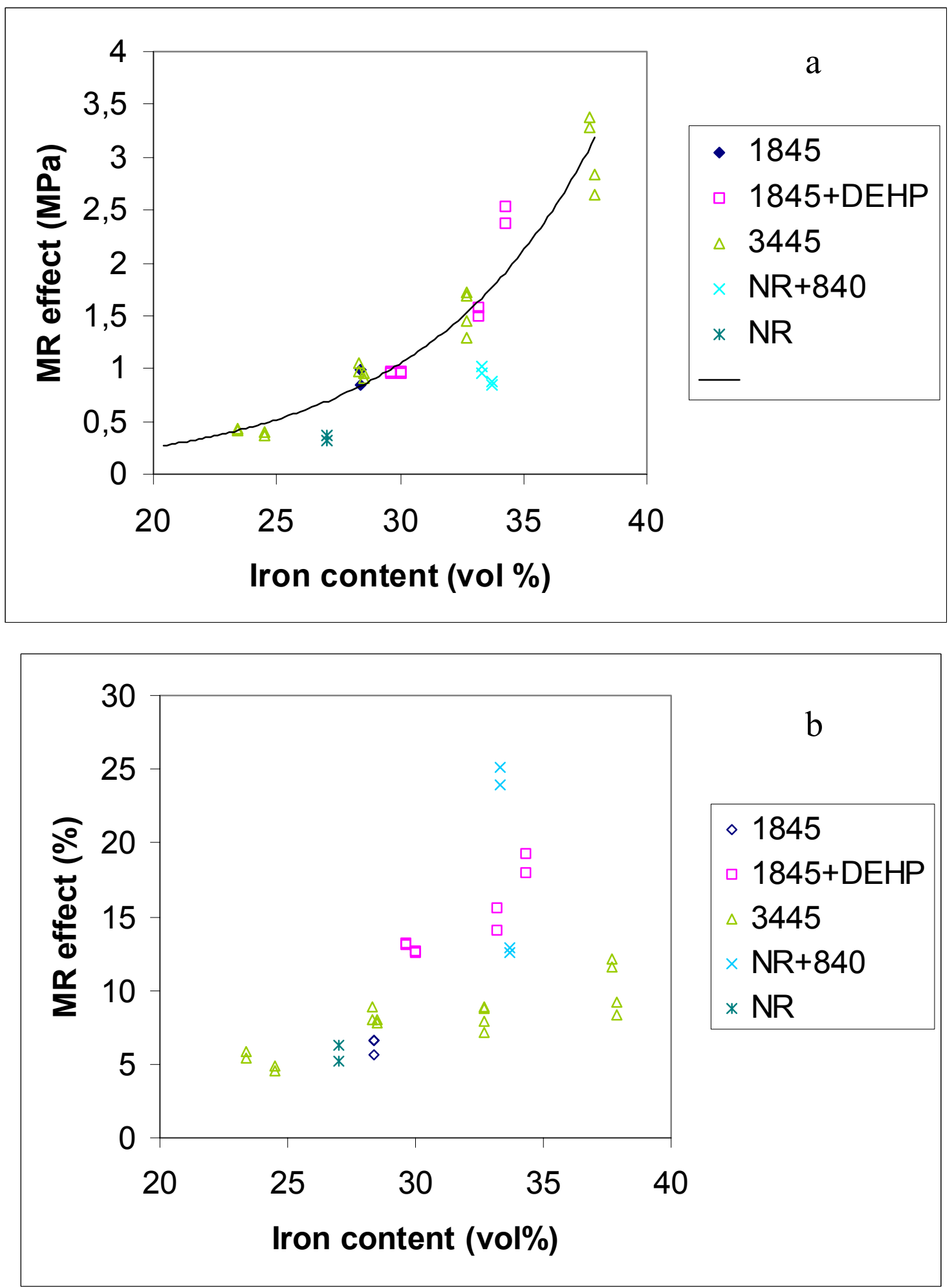

Figure 3.10. (a) Absolute and (b) relative MR Effect as functions of iron concentration for materials with different matrices. 


\subsection{MR Effect within the Audible Frequency Range}

The absolute MR effect in Perbunan 1845 with $32.4 \%$ by volume of ASC300 is shown in Figure 3.11 versus the frequency, 100 to $1250 \mathrm{~Hz}$ at magnetic inductions of $0,0.3,0.55$ and $0.8 \mathrm{~T}$. The results are similar To them obtained from measurements at low frequencies. The material is magnetically saturated at $0.5 \mathrm{~T}$, so the MR effect is not increased by applying stronger magnetic fields. An interesting feature of the results from the high frequency measurements is that the relative increase in the shear modulus is constant, about $18 \%$, over most of the frequency range, $400-1250 \mathrm{~Hz}$ (Figure 3.12). This MR effect is not sufficient for vibration isolation applications, but it can easily be increased by plasticising the matrix and increasing the iron content.

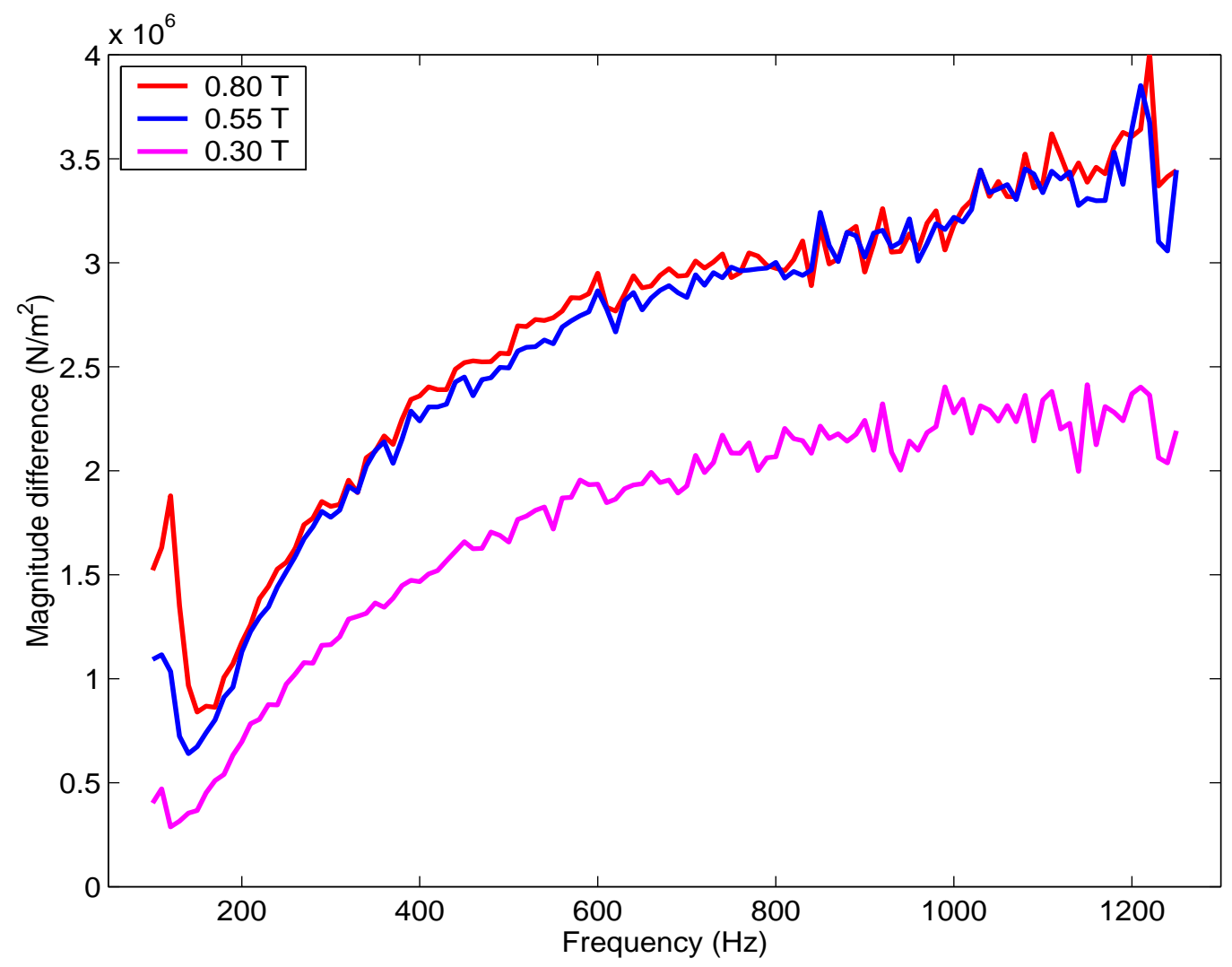

Figure 3.11 Absolute MR effect at high frequencies 


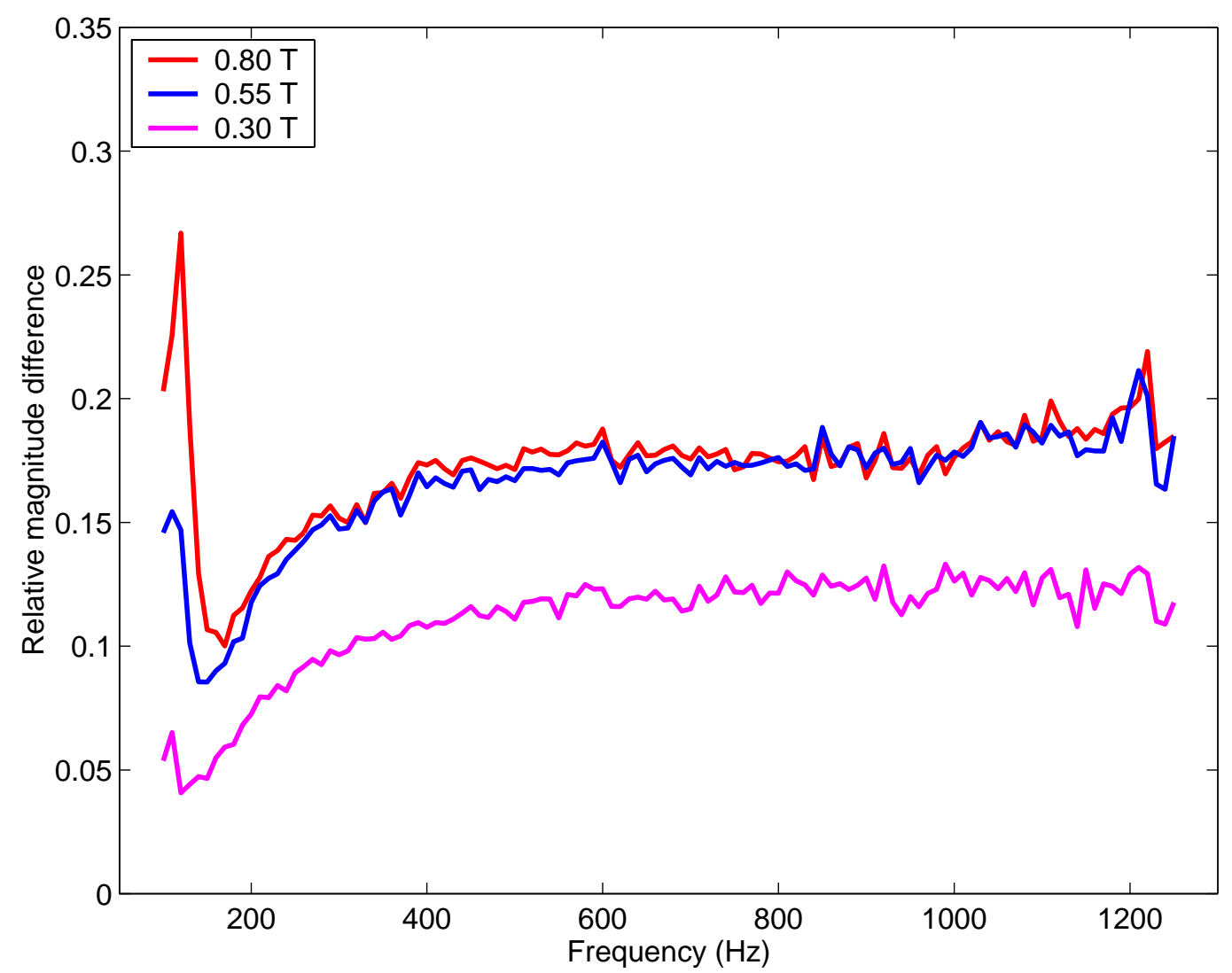

Figure 3.12. Relative MR effect at high frequencies.

The damping, $\tan \delta$, is almost independent of both the frequency and the applied magnetic field (Figure 3.13). It displays a value of about 0.2 at any applied magnetic field, over the whole frequency range. This is rather different from the results obtained at lower fields. In figure 3.14, $\tan \delta$ is shown for Perbunan 3445 with about $37 \%$ by volume of iron, at low frequencies $(1-21 \mathrm{~Hz})$. In this case, there is a small increase, about 0.01 0.02 , in the damping, due to the applied magnetic field. This result is similar to the results reported by Jolly et al. [17]. However, such a small increase in tan $\delta$ is of no practical importance. 


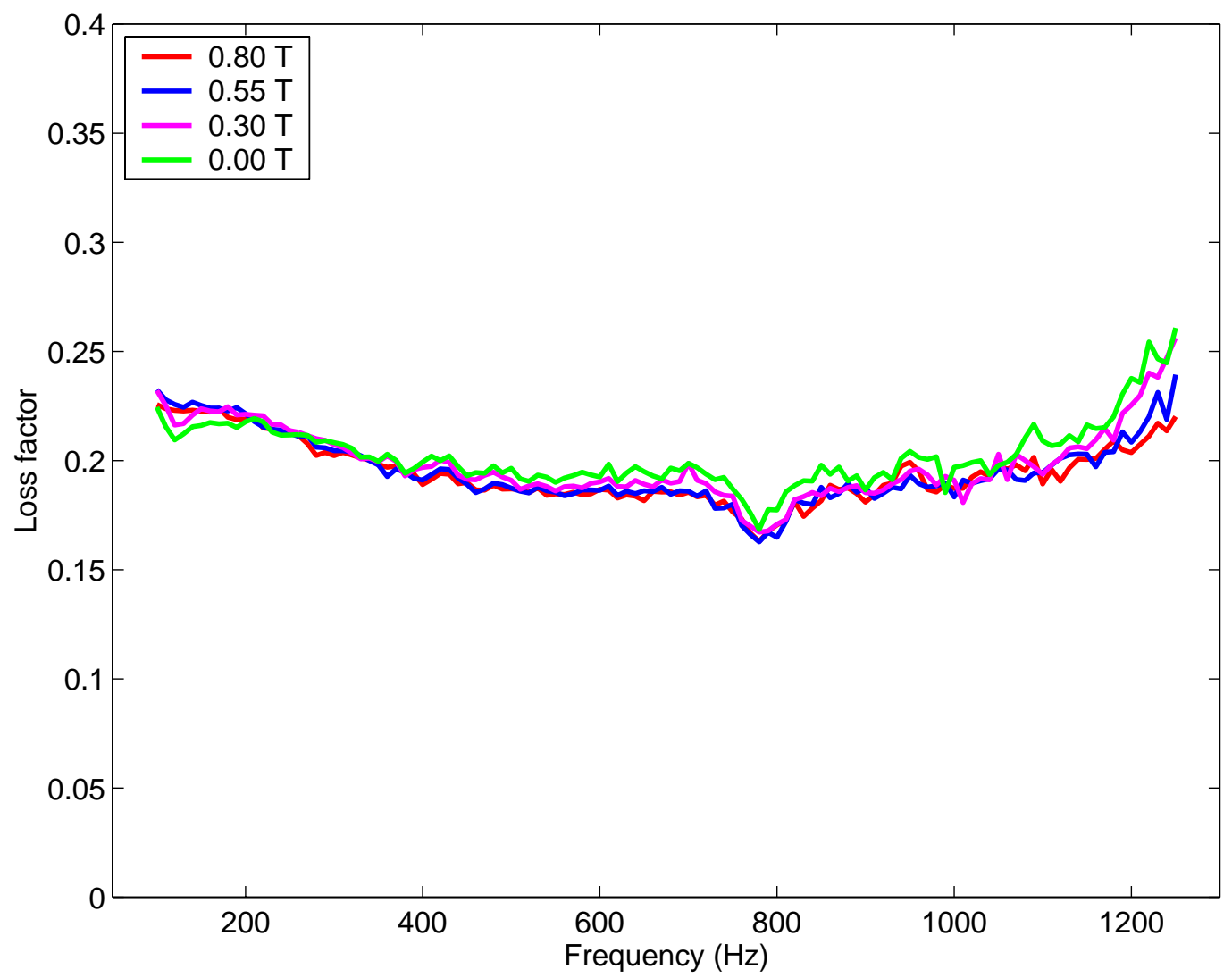

Figure 3.13 Damping (tan $\delta$ ) at high frequencies.

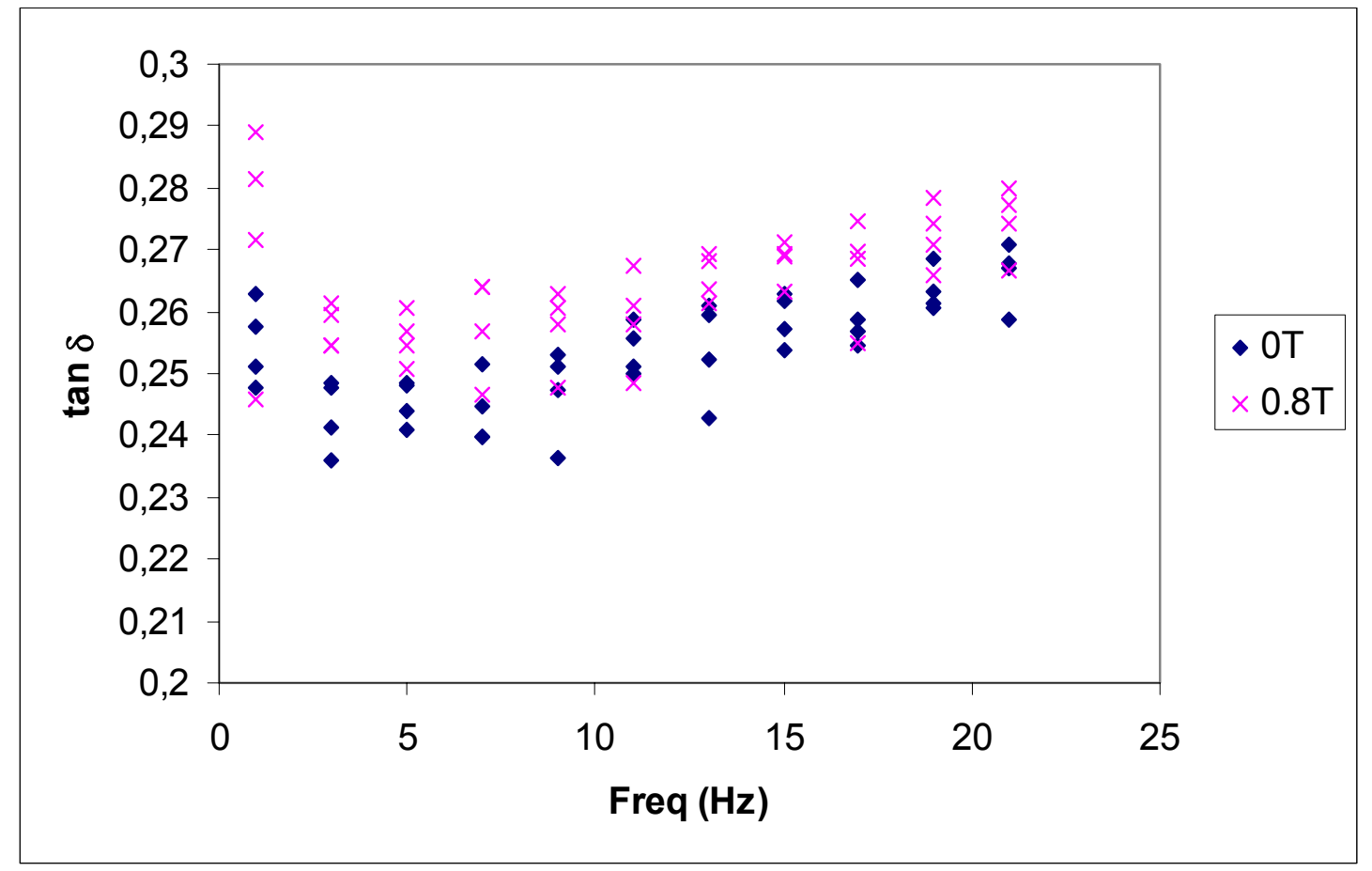

Figure 3.14. Damping of Perbunan 3445 with 37.8\% by volume of ASC300, measured without, and with an applied magnetic field of approximately $0.8 \mathrm{~T}$. 


\section{Conclusions}

Isotropic MR rubber materials with irregularly shaped iron particles exhibit a larger MR effect than isotropic MR rubber materials containing carbonyl iron. This large MR effect can be explained by the small distances between the particles, due to the low Critical Particle Volume Concentration (CPVC) of the irregular powders. Materials with poorly dispersed carbonyl iron, which results in aggregates of particles, exhibit a MR effect similar to that of materials with larger irregular particles.

The absolute MR effect in the isotropic MR rubbers with irregular particles is larger than that previously reported for MR solids $[11,17,19,20]$. This may, to some extent, be explained by the strain amplitude dependence of the MR effect, and the small strains we have used for general measurements. Nevertheless, when the peak-to-peak strain is the same, the absolute MR effect in isotropic MR rubbers is about twice as large as the effect in anisotropic MR rubbers [20].

In order to obtain a good isotropic MR rubber, the iron particle concentration must be close to the CPVC, where the inter-particle distances are minimal and the pre-cure orientation of the particles will not thereby increase the absolute MR effect. However, materials with such high iron particle concentrations have a high zero-field modulus; hence, the relative effect is quite low. The zero-field modulus can be decreased, and the relative MR effect thereby increased by the addition of plasticisers, or by the use of softer matrix materials like silicone rubbers. The strong strain amplitude dependence of the MR effect suggests that isotropic MR rubber materials are most suitable for low amplitude applications, such as for sound and vibration isolation. High frequency measurements show that this is a promising application for MR rubber materials. Finally, $\tan \delta$ of isotropic MR materials is somewhat increased by an applied magnetic field at low frequencies, but the increase is too small to be of any practical importance. At high frequencies, the damping has no MR effect. 


\section{Suggestions for Future Work}

The materials investigated in this work are promising materials for applications where controllable stiffness will improve the performance. However, much work remains to be done before any commercial devices will be on the market.

One aspect that has hitherto been completely neglected is the long-term properties of the materials. The incorporation of large amounts of iron into the elastomer matrix will influence the creep and relaxation of the matrix, and may induce crack growth. Furthermore, iron may also influence the oxidation of the matrix. More knowledge is needed about all these subjects before it will be possible to use the materials in commercial devices. 


\section{Acknowledgements}

First, I would like to thank my supervisor Professor Bengt Stenberg for persuading me to start working with this project, for accepting me as his $\mathrm{PhD}$-student, and for his continuous support, enthusiasm and never-ending optimism.

The European Commission is gratefully acknowledged for financial support (Contract no: G5RD-CT-1999-00125).

All the people at the department are thanked for the good times, for not hesitating to discuss anything, to help out, or to take coffee-breaks in the basement during the afternoons. The administrative staff, especially Maria and Margareta, are thanked for their help with all sorts of problems. Fredrik, my room-mate, is thanked for rewarding discussions on magnetorheology, weird music, and Hammarby. The last part of the rubber band, Petter, is thanked for being one major reason for the good atmosphere in our group. The other three heads of the Monster; Stina, Calle, and Jovan are thanked for solving all the problems in the world and re-creating the Swedish language during lunches, coffee-breaks and "after work beers". Mats Johansson is thanked for presenting the concept on "Critical Pigment Volume Concentration" during the course of Coating Technology, and Björn is thanked for gathering "Polymerlistan" for a dinner every now and then.

Thanks are due to the people at the Marcus Wallenberg Laboratory for Sound and Vibration Research (MWL): Kent Lindgren for invaluable help with the MR measurements, Leif Kari for discussions, ideas, and his part of "paper 2", and Danilo for letting me use his workspace, and for adjusting the measurement fixtures whenever we needed it.

I thank Fredrik Stillesjö and Julius Krah at the department of Electric Power Engineering (EKC) for introducing me to the field of magnetism, and for helping me on items regarding magnetic fields.

All partners of "IND-DAMPERS" are thanked for the valuable discussions and good times at our meetings, and for not making the co-ordination of the project more difficult than necessary.

J. Anthony Bristow is thanked for the rapid and skilful linguistic corrections.

My parents, sisters, and grandparents are thanked for always being there for me, and for always believing in me. All my friends outside the department are thanked for being friends. Serafin, Muadib, Bröderna Boomslang, and all other great musicians I have played with during these years are thanked for the music. 


\section{References}

1. Winslow W. M., Method and means for translating electrical impulses into mechanical force, 1947, US Patent 2,417,850.

2. Winslow W. M., "Induced Fibration of Suspensions", J. Appl. Phys., 1949. 20: p. 1137-1140.

3. Rabinow J., "The Magnetic Fluid Clutch", AIEE Transactions, 1948. 67: p. 13081315.

4. Jolly M. R., Bender J.W., Carlson J. D.,, "Properties and Applications of Commercial Magnetorheological Fluids", J. Intel. Mater. Syst. Struct., 1999. 10(1): p. 5-13.

5. Muñoz B. C., Jolly M.R., Composites with Field Responsive Rheology, in Performance of Plastics, Brostow W., Editor. 2001, Carl Hanser Verlag: Munich. p. 553-574.

6. Filisko F. E., "ER V: The Present and the Future (Rapporteur's Comments)", Int. J. Mod. Phys. B, 1996. 10(23 \& 24): p. v-vii.

7. Weiss K. D., Carlson J.D., Coulter J. P.,, "Material Aspects of Electrorheological Systems", J. Intel. Mater. Syst. Struct., 1993. 4(1): p. 13-34.

8. Carlson J. D. What makes a good MR fluid? in 8th International Conference on Electrorheological Fluids and Magnetorheological Suspensions. 2001. Nice: World Scientific.

9. $\quad$ Rigbi Z., Jilkén L., "The response of an elastomer filled with soft ferrite to mechanical and magnetic influences", J. Magn. Magn. Mater., 1983. 37: p. 267276.

10. Shiga T., Okada A., Kurauchi T.,, "Electroviscoelastic Effect of Polymer Blends Consisting of Silicone Elastomer and Semiconducting Polymer Particles", Macromolecules, 1993. 26: p. 6958-6963.

11. Shiga T., Okada A., Kurauchi, T., "Magnetroviscoelastic Behaviour of Composite Gels", J. Appl. Polym. Sci., 1995. 58: p. 787-792.

12. Rigbi Z., Mark J.E., "Effects of a Magnetic Field Applied during the Curing of a Polymer Loaded with Magnetic Filler", J. Appl. Polym. Sci., 1985. 23: p. $1267-$ 1269.

13. Jin S., Tiefel T.H., Wolfe R., Sherwood R. C., Mottine J.J. Jr.,, "Optically Transparent, Electrically Conductive Composite Medium", Science, 1992. 255: p. 446-448.

14. Kim S.S., Jo S.B., Gueon K. I., Choi K. K., Kim J. M., Churn K. S.,, "Complex Permeability and Permittivity and Microwave Absorption of Ferrite-Rubber Composite in X-band Frequencies", IEEE Transactions on Magnetics, 1991. 27(6): p. 5462-5464.

15. Pinho M. S., Gregori M.L., Nunes R. C. R., Soares B. G.,, "Aging effect on the reflectivity measurements of polychloroprene matrices containing carbon black and carbonyl-iron powder", Polym. Degrad. Stab., 2001. 73(1): p. 1-5.

16. Dishovsky N., Ruskova K., Dodov N.,, "On the Correlation Between Electromagnetic Waves Absorption and Electrical Conductivity of Iron Powder Filled Nitrile Butadiene Rubber", Macromol. Symp., 2001. 169: p. 313-319. 
17. Jolly M. R., Carlson J.D., Muñoz B. C., Bullions T. A.,, "The Magnetoviscoelastic Response of Elastomer Composites Consisting of Ferrous Particles Embedded in Polymer Matrix", J. Intel. Mater. Syst. Struct., 1996. 7(11): p. 613-622.

18. Jolly M. R., Carlson J.D., Muñoz B. C.," "A model of the behaviour of magnetorheological materials", Smart Materials and Structures, 1996. 5(5): p. 607-614.

19. Ginder J. M., Nichols M.E., Elie L.D., Tardiff J. L., "Magnetorheological Elastomers: Properties and Applications", SPIE, 1999. 3675: p. 131-138.

20. Nichols M.E., Ginder J.M., Tardiff J. L., Elie L. D.,. The Dynamic mechanical behaviour of magnetorheological elastomers. in 156th ACS Rubber Division Meeting. 1999. Orlando, Florida.

21. Carlson J. D., Jolly M.R., "MR fluid, foam and elastomer devices", Mechatronics, 2000. 10: p. 555-569.

22. Margida A. J., Weiss K.D., Carlson J. D.,, "Magnetorheological materials based on iron alloy particles", Int. J. Mod. Phys. B, 1996. 10(23 \& 24): p. 3335-3341.

23. Phulé P. P., Mihalcin M.P., Genc S., "The role of the dispersed-phase remnant magnetization on the redispersibility of magnetorheological fluids", J. Mater. Res., 1999. 14(7): p. 3037-3041.

24. Ashour O., Kinder D., Giurgiutiu V., Rogers C.,, "Manufacturing and characterization of magnetorheological fluids", SPIE, 1997. 3040: p. 174-184.

25. Lemaire E., Meunier A., Bossis G., Liu J., Felt D., Bahtovoi P., Matoussevitch N., "Influence of the particle size on the rheology of magnetorheological fluids", $J$. Rheol., 1995.39(5): p. 1011-1020.

26. Kordonski W. I., Gorodkin S.R., Novikova Z. A.,. The influence of ferroparticle concentration and size on mr fluid properties. in 6th International Conference on Electro-Rheological Fluids, Magneto-Rheological Suspensions and their Applications. 1997. Yonezawa, Japan: World Scientific.

27. Davis L.C., "Model of magnetorheological elastomers", J. Appl. Phys., 1999. 85(6): p. 3348-3351. 